\title{
Evaluation of seminal plasma interleukin 17 in infertile males
}

\author{
Original Ahmed Abdel-Bary ${ }^{1}$, Dalia Elnilly', Nouran Abo khedr ${ }^{1}$, Walid Mohamed Alamiri' ${ }^{1}$. \\ Article \\ ${ }^{1}$ Departments of Dermatology, Venereology and Andrology and ${ }^{2}$ Clinical Pathology, Faculty \\ of Medicine, Alexandria University, Alexandria, Egypt
}

\begin{abstract}
Background: Interleukin-17 (IL-17) is a proinflammatory cytokine that is involved in the pathogenesis of several autoimmune and inflammatory diseases. Inflammation of the testis and male-reproductive tracts may lead to male infertility.

Objective: To assess seminal plasma IL-17 in infertile male patients and to evaluate its relation to the different seminal parameters.

Patients and Methods: Fifty male patients with primary infertility were included in addition to 20 age-matched healthy fertile males as controls. The semen was analyzed half an hour after the ejaculation according to the WHO criteria, including sperm count, morphology, motility, viability, and leukocytecount. Seminal plasma levels of IL-17 were measured using the ELISA technique.

Results: The mean seminal plasma level of IL-17 was significantly higher in infertile patients $(2.42 \pm 2.43 \mathrm{ng} / \mathrm{l})$ than in controls $(0.28 \pm 0.66 \mathrm{ng} / \mathrm{l})$. Also, the mean seminal plasma IL-17 level was significantly higher in patients with abnormal progressive sperm motility $(4.0 \pm 2.73 \mathrm{ng} / \mathrm{l})$ than patients with normal progressive sperm motility $(2.07 \pm 2.26 \mathrm{ng} / 1)$.

Conclusion: IL-17 may play an important role in the pathogenesis of male infertility associated with inflammation and infection of the testis and male genital tracts and it could be a good therapeutic target in those cases, but further studies are needed to determine the exact pathogenic role of IL-17 in male infertility.
\end{abstract}

Key Words: Interleukin 17, male infertility, seminal plasma.

Received: 17 June 2021, Accepted: 21 August 2021

Corresponding Author: Ahmed Abdel-Bary, Department of Dermatology, Venereology and Andrology, Faculty of Medicine, Alexandria University, Alexandria, Egypt, Tel.: 01282840574, E-mail: drahmedabdelbary2016@gmail.com

ISSN: 2090-6048, 2021

\section{INTRODUCTION}

Infertility is the inability of a couple to gain pregnancy after 1 year of regular unprotected intercourse, males are responsible for about $30-50 \%$ of the cases ${ }^{[1]}$. There are multiple causes of male infertility, including genetic abnormalities, hormonal disturbances, varicocele, infections, drugs, obstruction, and others ${ }^{[2]}$.

The testis is an immunoprivileged organ in which the anatomical blood-testis barrier and the local immunosuppressive environment act together to protect immunogenic germ cells from systemic immune attack, at the same time, effective local innate immunity protects the testis from infective pathogens ${ }^{[3]}$. Inflammation of the testis and male-reproductive tracts due to infections, varicocele, ejaculatory-duct obstructions, or testicular torsion may lead to disruption of this immunoprivilege, testicular dysfunction, and infertility ${ }^{[4]}$.

Different cytokines are secreted under normal conditions in the male gonads, they are responsible for the regulation of spermatogenesis and steroidogenesis, but on the other hand, inflammatory cytokines in cases of testicular infection and inflammation are harmful ${ }^{[5]}$.

Interleukin-17 (IL-17) is a proinflammatory cytokine produced mainly by T-helper 17 cells. It has been associated with multiple autoimmune and inflammatory conditions, such as rheumatoid arthritis, psoriasis, Crohn's disease, and systemic lupus erythematosus, but its role in male infertility is still understudied ${ }^{[6]}$. So, we aimed to assess seminal plasmaIL-17 in infertile male patients and to evaluate its relation to the different seminal parameters.

\section{PATIENTS AND METHODS}

Fifty male patients with primary infertility were selected for this case-control study from the Outpatient Clinic of the Dermatology, Venereology and Andrology Department, Alexandria Main University Hospital, Egypt, in addition, 20 age-matched healthy fertile males were included as controls.

Patients having diseases that may increase serum IL-17, such as rheumatoid arthritis, multiple sclerosis, Behcet disease, systemic lupus erythematosus, systemic sclerosis psoriasis, atopic dermatitis, and hidradenitis 
suppurativa, were excluded from this study.

Patient's consent was taken and the study was approved by the local Ethics Committee at the Faculty of Medicine, University of Alexandria, Egypt.

Patients were subjected to full history taking focusing on childhood illnesses such as viral orchitis or cryptorchidism, genital trauma, and prior pelvic or inguinal surgery, infections such as epididymitis or urethritis, drugs, smoking, radiation therapy, and chemotherapy.

Complete physical and genital examination was done with emphasis on the secondary sexual characteristics, size and consistency of the testicles, presence or absence of vas, and presence or absence of a varicocele.

\section{Semen analysis}

Semen was collected from the patients and controls after 4-5 days of abstinence. The semen was analyzed half an hour after the ejaculation according to the WHO criteria, including sperm count, morphology, motility, viability, and leukocyte count ${ }^{[7]}$.

\section{Measurement of seminal plasma interleukin-17 level}

The collected semen sample was centrifuged for 20 $30 \mathrm{~min}$ at a speed of 2000-3000 rounds per minute, and then the supernatant was removed and if the precipitate appeared, we centrifuged the sample again.

Seminal plasma levels of IL-17 were measured using the ELISA technique. HumanInterleukin-17 ELISA Kit (Cat. No SG-10278 provided by Sinogeneclon Biotech Co. Ltd, Hangzhou, China) was used. The plate has been precoated with human IL-17 antibody, IL-17 present in the sample is added and binds to antibodies coated on the wells. Then, human IL-17 antibody is added and binds to IL-17 in the sample. Then streptavidin-HRP is added and binds to the IL-17 antibody. After incubation, unbound streptavidinHRP is washed away during a washing step. Substrate solution is then added and color develops in proportion to the amount of human IL-17. The concentration of IL-17 in the samples is then determined by comparing the OD of the samples with the standard curve.

\section{Statistical analysis}

Data were fed to the computer and analyzed using IBM SPSS software package, version 20.0(IBM Corp., Armonk, New York, USA).Qualitative data were described using number and percent. The Kolmogorov-Smirnov test was used to verify the normality of distribution. Quantitative data were described using range (minimum and maximum), mean, SD, median, and interquartile range. Significance of the obtained results was judged at the $5 \%$ level.

The used tests were $\chi^{2}$ test for categorical variables, to compare between different groups, Fisher's exact or Monte Carlo correction for $\chi^{2}$ when more than $20 \%$ of the cells have expected count less than 5,Mann-Whitney test for abnormally distributed quantitative variables, to compare between two studied groups, Spearman coefficient test to correlate between two distributed abnormally quantitative variables, and Kruskal-Wallis test for abnormally distributed quantitative variables.

\section{RESULTS}

The mean age of the studied patients was $27.70 \pm 18.54$ years, $39(78 \%)$ patients had varicocele, whereas leukocytospermia $\left(\geq 1 \times 10^{6}\right.$ leukocytes $/ \mathrm{ml}$ of seminal plasma) was found in $15(30 \%)$ patients.

\section{Sperm concentration}

Out of 50 infertile patients, 27 (54\%) patients were oligospermic and $13(26 \%)$ patients were azoospermic and the remaining $10(20 \%)$ patients had normal sperm count.

\section{Sperm motility}

Thirty-five $(70 \%)$ patients had abnormal progressive motility and $15(30 \%)$ patients had normal progressive motility.

\section{Sperm morphology}

Normal sperm morphology was found in 23(46\%) patients and abnormal sperm morphology was found in 27 (54\%) patients.

\section{Sperm viability}

Normal sperm viability $(\geq 58 \%)$ was present in 21 patients and abnormal viability was present in 29 patients.

Table 1 clarifies the main seminal parameters among the patients and the controls.

\section{Seminal plasma level of interleukin-17}

The mean seminal plasma level of IL-17was significantly higher in infertile patients $(2.42 \pm 2.43 \mathrm{ng} / \mathrm{l})$ than in controls $(0.28 \pm 0.66 \mathrm{ng} / \mathrm{l})(<0.001)$ (Table 2$)$. 
Table 1: The sperm concentration, motility and morphology among cases and control

\begin{tabular}{|c|c|c|c|c|}
\hline & Cases $(\mathrm{N}=50)[\mathrm{n}(\%)]$ & Controls $(\mathrm{N}=50)[\mathrm{n}(\%)]$ & Test of significance & $P$ \\
\hline \multicolumn{5}{|l|}{ Sperm concentration } \\
\hline Normal $\left(\geq 1 \times 10^{6} / \mathrm{ml}\right)$ & $10(20.0)$ & $20(100.0)$ & \multirow{4}{*}{$\chi 2=37.333^{*}$} & \multirow{4}{*}{$<0.001^{*}$} \\
\hline Oligospermia & $27(54.0)$ & 0 & & \\
\hline Azospermia & $13(26.0)$ & 0 & & \\
\hline Minimum-maximum & $0.0-59.10$ & $15.40-168.0$ & & \\
\hline Mean \pm SD & $9.70 \pm 13.86$ & $56.34 \pm 42.25$ & \multirow[t]{2}{*}{$\mathrm{U}=64.00 *$} & \multirow[t]{2}{*}{$<0.001^{*}$} \\
\hline Median (IQR) & $4.75(0.0-11.50)$ & $42.80(27.25-74.50)$ & & \\
\hline \multicolumn{5}{|l|}{ Progressive motility } \\
\hline Normal ( $\geq 32 \%)$ & $15(30.0)$ & $20(100.0)$ & \multirow{3}{*}{$\chi 2=28.00 *$} & \multirow{3}{*}{$<0.001 *$} \\
\hline Abnormal & $35(70.0)$ & 0 & & \\
\hline Minimum-maximum & $0.0-59.0$ & $38.50-62.50$ & & \\
\hline Mean \pm SD & $18.64 \pm 19.47$ & $50.39 \pm 8.14$ & \multirow[t]{2}{*}{$\mathrm{U}=95.00 *$} & \multirow[t]{2}{*}{$<0.001^{*}$} \\
\hline Median (IQR) & $11.0(0.0-33.50)$ & $48.55(44.90-60.15)$ & & \\
\hline \multicolumn{5}{|l|}{ Sperm morphology } \\
\hline Normal $(\geq 4 \%)$ & $23(46.0)$ & $23(46.0)$ & \multirow{3}{*}{$\chi 2=17.581 *$} & \multirow{3}{*}{$<0.001^{*}$} \\
\hline Abnormal & $27(54.0)$ & $27(54.0)$ & & \\
\hline Minimum-maximum & $0.0-83.0$ & $0.0-83.0$ & & \\
\hline Mean \pm SD & $12.18 \pm 22.91$ & $12.18 \pm 22.91$ & \multirow[t]{2}{*}{$\mathrm{U}=270.50^{*}$} & \multirow[t]{2}{*}{$0.003 *$} \\
\hline Median (IQR) & $2.75(0.0-8.0)$ & $2.75(0.0-8.0)$ & & \\
\hline
\end{tabular}

$\chi 2, \chi 2$ test; IQR, interquartile range; U,Mann-Whitney test.

* Statistically significant at $P$ value less than or equal to 0.05 .

Table 2: Comparison between patients and control according to interleukin-17 seminal plasma level (ng/l)

\begin{tabular}{lccc}
\hline IL-17 & Cases $(\mathrm{N}=50)$ & Control $(\mathrm{N}=20)$ & U \\
\hline Minimum-maximum & $0.0-8.75$ & $0.0-2.34$ & $P$ \\
Mean \pm SD & $2.42 \pm 2.43$ & $0.28 \pm 0.66$ & $159.50^{*}$ \\
Median (IQR) & $1.83(0.37-4.24)$ & $0.0(0.0-0.12)$ & $<0.001^{*}$ \\
\hline
\end{tabular}

IL, interleukin-17; IQR, interquartile range; U, Mann-Whitney test.

*Statistically significant at $P$ value less than or equal to 0.05

Relation between seminal plasma interleukin-17 level and seminal parameters among the studied patients

No significant correlation could be detected between seminal plasma IL-17 level and sperm concentration, but azoospermic patients had the highest mean seminal plasma IL-17 level $(2.89 \pm 2.27 \mathrm{ng} / \mathrm{l})($ Table 3$)$.

Also, there was no significant correlation between seminal plasma IL-17 level and each of sperm morphology and sperm viability ( $P=0.246$ and 0.898 , respectively) (Table 3).
The mean seminal plasma IL-17 level was significantly higher in patients with abnormal progressive sperm motility $(4.0 \pm 2.73 \mathrm{ng} / 1)$ than patients with normal progressive sperm motility $(2.07 \pm 2.26 \mathrm{ng} / \mathrm{l})$. Also, it was significantly higher in patients with leukocytospermia $(3.06 \pm 2.43 \mathrm{ng} / \mathrm{l})$ than those with normal semen leukocyte count $(0.39 \pm 0.82 \mathrm{ng} / \mathrm{l})$ (Mann-Whitney test, $\mathrm{U}=51.50, P \leq 0.001$ ).

No significant difference could be detected between the seminal plasma IL-17 level in patients with or without varicocele (Mann-Whitney test, $\mathrm{U}=217.50, P=0.610$ ). 
Table 3: Relation between interleukin-17 and different seminal parameters in patients $(\mathrm{N}=50)$

\begin{tabular}{|c|c|c|c|c|c|c|}
\hline \multicolumn{7}{|c|}{ IL-17 } \\
\hline & $\mathrm{N}$ & Minimum-maximum & Mean \pm SD & Median & Test of significance & $P$ \\
\hline \multicolumn{7}{|c|}{ Sperm concentration } \\
\hline Normal & 10 & $0.0-6.98$ & $2.13 \pm 2.45$ & 0.92 & \multirow{3}{*}{$\mathrm{H}=1.380$} & \multirow{3}{*}{0.502} \\
\hline Oligospermia & 27 & $0.0-7.46$ & $2.56 \pm 2.74$ & 1.93 & & \\
\hline Azospermia & 13 & $0.0-8.75$ & $2.89 \pm 2.27$ & 2.20 & & \\
\hline \multicolumn{7}{|c|}{ Progressive motility } \\
\hline Normal & 15 & $0.0-8.75$ & $2.07 \pm 2.26$ & 1.37 & \multirow{2}{*}{$\mathrm{U}=106.0^{*}$} & \multirow{2}{*}{$0.048^{*}$} \\
\hline Abnormal & 35 & $0.27-7.46$ & $4.0 \pm 2.73$ & 4.24 & & \\
\hline \multicolumn{7}{|c|}{ Sperm morphology } \\
\hline Normal & 23 & $0.0-6.80$ & $2.0 \pm 2.22$ & 1.08 & \multirow{2}{*}{$\mathrm{U}=251.0$} & \multirow{2}{*}{0.246} \\
\hline Abnormal & 27 & $0.0-8.75$ & $2.77 \pm 2.59$ & 2.20 & & \\
\hline \multicolumn{7}{|l|}{ Viability } \\
\hline Normal & 21 & $0.0-7.46$ & $2.39 \pm 2.38$ & 1.08 & \multirow{2}{*}{$\mathrm{U}=298.0$} & \multirow{2}{*}{0.898} \\
\hline Abnormal & 29 & $0.0-8.75$ & $2.46 \pm 2.56$ & 1.87 & & \\
\hline
\end{tabular}

H, Kruskal-Wallis test; U, Mann-Whitney test.

* Statistically significant at $P$ value less than or equal to 0.05 .

\section{DISCUSSION}

Cytokines are important molecules in the communication between the different cells in the bodyin both normal and pathological conditions ${ }^{[8]}$. Different cytokines like IL-1, IL-6, tumor necrosis factor- $\alpha$, and transforming growth factor- $\beta$ had been found to be secreted by Leydig cells and Sertoli cells under physiological conditions in the normal testicles and it had been shown that they are involved in the regulation of testicular function ${ }^{[9]}$.

On the other hand, testicular inflammation and infection lead to increased secretion of proinflammatory cytokines, which may affect spermatogenesis and fertility ${ }^{[10]}$.

In our study, seminal plasma IL-17 level in infertile patients was significantly higher in comparison with healthy controls, which sheds light on its role in the pathogenesis of male infertility. In agreement with our results, Babinets et $a l^{[11]}$ found that the concentration of IL-17 increases in infertile men with chronic urethral prostatitis and they concluded that it may be a good biomarker of chronic inflammation of the urogenital tract.

Duan et al. ${ }^{[12]}$ studied the expression of T-helper 17 cells in human azoospermic testis with chronic inflammation and concluded that their high expression in those patients highlights the role of IL-17 in male infertility through disruption of testicular function.

In order to evaluate the pathogenic role of IL-17, Pérez et $a l^{[13]}$ induced experimental autoimmune orchitis in rats and found that IL-17 can impair the testicular bloodtestis barrier and facilitate the recruitment of immune cells to the testicular interstitium and induce sloughing and apoptosis of the germinal epithelium.

Negative correlation between the seminal plasma IL-17 level and sperm motility had been observed in our study, similar results had been reported by Qian et al. ${ }^{[14]}$, it is thought that increased oxidative stress in seminal plasma induced by different cytokines could be the mechanism by which IL-17could impair sperm motility ${ }^{[15]}$.

The significant increase in seminal plasma IL-17 level in patients with leukocytospermia observed in our study may shed light on the role of IL-17 in the pathogenesis of infertility induced by urogenital infection. Hakimi et al. ${ }^{[16]}$ observed elevated expressions of IL-17 in patients with chlamydia trachomatis infection.

\section{CONCLUSION}

It could be concluded that IL-17 may play an important role in the pathogenesis of male infertility associated with inflammation and infection of the testis and male genital tracts and it could be a good therapeutic target in those cases, but further studies are needed to determine the exact pathogenic role of IL-17 in male infertility.

\section{CONFLICT OF INTEREST}

There are no conflicts of interest. 


\section{REFERENCES}

1. Katz D J, Teloken P, Shoshany O. Male infertility - the other side of the equation. Aust Fam Physician2017; 46:641-646.

2. Naz M, Kamal M. Classification, causes, diagnosis and treatment of male infertility: a review. Orient Pharm Exp Med2017; 17:89-109.

3. Zhao S, Zhu W, Xue S, Han D. Testicular defense systems: immune privilege and innate immunity. Cell Mol Immunol2014; 11:428-437.

4. Azenabor A, Ekun A O, Akinloye O. Impact of inflammation on male reproductive tract. J Reprod Infertil2015; 16:123-129.

5. Syriou V, Papanikolaou D, Kozyraki A, Goulis D G. Cytokines and male infertility. Eur Cytokine Netw2018; 29:73-82.

6. Shabgah A G, Fattahi E, Shahneh F Z. Interleukin-17 in human inflammatory diseases.Postepy Dermatol Alergol2014; 31:256-261.

7. WHO laboratory manual for the examination and processing of human semen, fifth edition. Geneva: World Health Organization; 2010.

8. Dinarello C A. Historical insights into cytokines. Eur J Immunol2007; 37:34-45.

9. Loveland K L, Klein B, Pueschl D, Indumathy S, Bergmann M, Loveland B E, Hedger M P, Schuppe $\mathrm{H}$ C. Cytokines in male fertility and reproductive pathologies: immunoregulation and beyond. Front Endocrinol 2017; 8:307.
10. Irez $T$, Bicer $S$, Sahin $E$, Dutta $S$, Sengupta $P$. Cytokines and adipokines in the regulation of spermatogenesis and semen quality. Chem Biol Lett2020; 7:131-139.

11. Babinets L S, Migenko B O, Borovyk I O, Halabitska I M, Lobanets $\mathrm{N} \mathrm{V}$, Onyskiv $\mathrm{O} \mathrm{O}$. The role of cytokine imbalance in the development of man infertility. Wiad Lek2020; 73:525-528.

12. Duan Y G, Yu C F, Novak N, Bieber T, Zhu C H, Schuppe H C, Haidl G, Allam J P. Immunodeviation towards a Th17 immune response associated with testicular damage in azoospermic men. Int $\mathrm{J}$ Androl2011; 34(6pt2):e536-e545.

13. Pérez C V, Pellizzari E H, Cigorraga S B, Galardo M N, Naito M, Lustig L, Jacobo P V. IL17A impairs blood-testis barrier integrity and induces testicular inflammation. Cell Tissue Res2014;358:885-898.

14. Qian L, Shi Q, Gu Y, Song J, Zhou M, Hua M. The relationship between IL-17 and male infertility: semen analysis. Afr J Microbiol Res 2012; 6:5672-5677.

15. Kurkowska W, Bogacz A, Janiszewska M, Gabrys E, Tiszler M, Bellanti $\mathrm{F}$, et al.Oxidative stress is associated with reduced sperm motility in normal semen. Am J Mens Health 2020; 14:1557988320939731

16. Hakimi H, Akhondi M M, Sadeghi M R, Chamani L, Arababadi M K, Ahmadabadi B N, Hassanshahi G, Fathollahi M S.Seminal levels of IL-10, IL-12, and IL-17 in men with asymptomatic chlamydia infection. Inflammation2014; 37:122-126. 